PoS $\quad \begin{aligned} & \text { PROCEEDINGS } \\ & \text { OF SCIENCE }\end{aligned}$

\title{
NNLO evolution equation for flavor-nonsinglet operators in off-forward kinematics
}

\author{
Alexander Manashov* \\ Hamburg University, Hamburg, GERMANY \\ E-mail: alexander.manashov@desy.de
}

We derive the NNLO evolution equation in non-forward kinematic for flavor-nonsinglet leading twist operators in the $\overline{\mathrm{MS}}$ scheme. Results were obtained both for evolution kernel for lightray operators and the anomalous dimension matrix for local operators. They are also directly applicable to the renormalization of the pion light-cone distribution amplitude.

13th International Symposium on Radiative Corrections

24-29 September, 2017

St. Gilden, Austria

${ }^{*}$ Speaker. 


\section{Introduction}

Scale dependence of physical observables in processes involving a large momentum transfer is governed by the anomalous dimensions of the corresponding leading twist operators. In order to make the theory description fully quantitative they have to be calculated to a sufficiently high order in perturbation theory. At present, the anomalous dimensions of the twist-two operators are known to NNLO accuracy (three loops) $[1,2]$. These results are used in modern description of inclusive reactions, e.g., at the $\mathrm{LHC}$.

A remarkable progress in accelerator technologies in the last decades has made possible the study of hard exclusive reactions with identified particles in the final state. The theoretical description of such reactions involves operator matrix elements between states with different momenta as the nonperturbative input. The renormalization group equations (RGEs) for such matrix elements are more complicated since one has to take into account the mixing with the operators involving total derivatives. The mixing matrix has a triangular form with the diagonal entries being the anomalous dimensions. Nevertheless the off-diagonal contributions require a dedicated calculation.

A direct calculation in higher orders is rather complicated. However, tt has been known for some time [3] that the form of mixing matrix is greatly constrained by the conformal symmetry. The full evolution kernel at given order of perturbation theory is completely determined by the socalled special conformal anomaly at one order less. This result was used to compute the two-loop mixing matrix for twist-two operators in QCD $[4,5,6]$ and obtain the two-loop evolution kernels in non-froward kinematics $[7,8,9]$.

An alternative technique was developed in Ref. [10]. Instead of studying conformal symmetry breaking effects in the physical theory $[4,5,6]$ it was suggested to use the exact conformal symmetry of a modified theory - QCD in $d=4-2 \varepsilon$ dimensions at critical coupling. Utility of this modified approach was demonstrated in [10] on several examples for scalar theories, and, in $[11,12,13]$ for flavor-nonsinglet operators in QCD.

The calculation of three-loop evolution kernel for flavor-nonsinglet operators [13] is summarized below.

\section{Evolution equations for light-ray operators}

A renormalized light-ray operator [14] serves as the generating function for renormalized local operators (in what follows we always assume $\overline{\mathrm{MS}}$ scheme)

$$
[\mathscr{O}]\left(x ; z_{1}, z_{2}\right)=Z \bar{q}\left(x+z_{1} n\right) h q\left(x+z_{2} n\right)=\sum_{m, k} \frac{z_{1}^{m} z_{2}^{k}}{m ! k !}\left[\bar{q}(x)(\overleftarrow{D} \cdot n)^{m} \not h(n \cdot \vec{D})^{k} q(x)\right] .
$$

The renormalization factor $Z$ in this formulation is an integral operator in $z_{1}, z_{2}$ which is given by a power series in $1 / \varepsilon$

$$
Z=1+\sum_{k=0}^{\infty} \varepsilon^{-k} Z_{k}(a), \quad Z_{k}(a)=\sum_{\ell=k}^{\infty} a^{\ell} Z_{k}^{(\ell)} .
$$

The renormalized light-ray operator satisfies the RGE

$$
\left(M \partial_{M}+\beta(a) \partial_{a}+\mathbb{H}(a)\right)[\mathscr{O}]\left(x ; z_{1}, z_{2}\right)=0,
$$


where $M$ is the renormalization scale, $a=\alpha_{s} / 4 \pi$,

$$
\beta(a)=M \frac{d a}{d M}=-2 a\left(\varepsilon+a \beta_{0}+a^{2} \beta_{1}+\ldots\right)=-2 a(\varepsilon+\bar{\beta}(a)), \quad \beta_{0}=\frac{11}{3} N_{c}-\frac{2}{3} n_{f}
$$

and $\mathbb{H}(a)$ is an integral operator acting on the light-cone coordinates of the fields. It has the following form

$$
\mathbb{H}(a)=a \mathbb{H}^{(1)}+a^{2} \mathbb{H}^{(2)}+a^{3} \mathbb{H}^{(3)}+\ldots
$$

The evolution kernel can be written as

$$
[\mathbb{H}(a) f]\left(z_{1}, z_{2}\right)=\int_{0}^{1} d \alpha \int_{0}^{1} d \beta h(\alpha, \beta) f\left(z_{12}^{\alpha}, z_{21}^{\beta}\right),
$$

where $z_{12}^{\alpha}=z_{1} \bar{\alpha}+z_{2} \alpha$ and $\bar{\alpha}=1-\alpha$. The translation-invariant polynomials $\left(z_{1}-z_{2}\right)^{N}$ are eigenfunctions of the evolution kernel,

$$
\mathbb{H}(a) z_{12}^{N}=\gamma_{N}(a), z_{12}^{N} \quad z_{12}=z_{1}-z_{2}
$$

with the eigenvalues $\gamma_{N}(a)$ given by moments of the evolution kernel in the representation (2.6),

$$
\gamma_{N}=\int_{0}^{1} d \alpha \int_{0}^{1} d \beta(1-\alpha-\beta)^{N} h(\alpha, \beta)
$$

The leading order kernel $\mathbb{H}^{(1)}$ commutes with the canonical generators of collinear conformal transformations

$$
S_{-}^{(0)}=-\partial_{z_{1}}-\partial_{z_{2}}, \quad S_{0}^{(0)}=z_{1} \partial_{z_{1}}+z_{2} \partial_{z_{2}}+2, \quad S_{+}^{(0)}=z_{1}^{2} \partial_{z_{1}}+z_{2}^{2} \partial_{z_{2}}+2\left(z_{1}+z_{2}\right),
$$

which obey the standard $S L(2)$ commutation relations

$$
\left[S_{0}, S_{ \pm}\right]= \pm S_{ \pm}, \quad\left[S_{+}, S_{-}\right]=2 S_{0}
$$

As a consequence of the commutation relations $\left[\mathbb{H}^{(1)}, S_{\alpha}^{(0)}\right]=0$ the evolution kernel $h^{(1)}(\alpha, \beta)$ is effectively a function of one variable $\tau=\alpha \beta / \bar{\alpha} \bar{\beta}$ which is called the conformal ratio [15], $h^{(1)}(\alpha, \beta)=\bar{h}(\tau)$. This function is completely determined by its moments (2.8).

This property is lost beyond the leading order. Nevertheless, one could expect that in a conformal theory, such as QCD in $d=4-2 \varepsilon$ dimensions at the critical point $a=a_{*}\left(\beta\left(a_{*}\right)=0\right)$ in particular, the evolution kernel commutes with the generators of conformal transformations. These generators of course differ from their canonical form due to quantum corrections. In the $\overline{\mathrm{MS}}$ scheme the evolution kernel does not depend on the space-time dimension. As a consequence, the evolution kernels in QCD in four dimensions are exactly the same as in QCD in $d=4-2 \varepsilon$ dimensions. Therefore the QCD evolution kernel $\mathbb{H}(a)$ commutes with three operators $[10,11,12]$

$$
\left[\mathbb{H}(a), S_{+}\right]=\left[\mathbb{H}(a), S_{-}\right]=\left[\mathbb{H}(a), S_{0}\right]=0
$$


that satisfy the $S L(2)$ algebra (2.10). These operators were constructed in [12] and have the following form

$$
\begin{aligned}
& S_{-}=S_{-}^{(0)} \\
& S_{0}=S_{0}^{(0)}+\Delta S_{0}=S_{0}^{(0)}+\left(\bar{\beta}(a)+\frac{1}{2} \mathbb{H}(a)\right) \\
& S_{+}=S_{+}^{(0)}+\Delta S_{+}=S_{+}^{(0)}+\left(z_{1}+z_{2}\right)\left(\bar{\beta}(a)+\frac{1}{2} \mathbb{H}(a)\right)+\left(z_{1}-z_{2}\right) \Delta(a) .
\end{aligned}
$$

Here $\bar{\beta}(a)$ is the QCD $\beta$-function (2.4) and $S_{\alpha}^{(0)}$ are the canonical generators (2.9). The form of the generators $S_{-}$and $S_{0}$ is fixed from general consideration while $S_{+}$requires a dedicated calculation.

Since $\left[S_{-}, \mathbb{H}(a)\right]=\left[S_{0}, \mathbb{H}(a)\right]=0$ the only nontrivial relation on the evolution kernel comes from the last commutator $\left[\mathbb{H}(a), S_{+}\right]=0$. It results in the following relation

$$
\left[S_{+}^{(0)}, \mathbb{H}(a)\right]=\left[\mathbb{H}(a), z_{1}+z_{2}\right]\left(\bar{\beta}(a)+\frac{1}{2} \mathbb{H}(a)\right)+\left[\mathbb{H}(a), z_{12} \Delta(a)\right]
$$

Expanding the kernels in a power series in coupling constant

$$
\mathbb{H}(a)=a \mathbb{H}^{(1)}+a^{2} \mathbb{H}^{(2)}+a^{3} \mathbb{H}^{(3)}+\ldots, \quad \Delta(a)=a \Delta^{(1)}+a^{2} \Delta^{(2)}+\ldots
$$

one obtains from (2.13) a nested set of equations [10]

$$
\begin{aligned}
{\left[S_{+}^{(0)}, \mathbb{H}^{(1)}\right]=} & 0 \\
{\left[S_{+}^{(0)}, \mathbb{H}^{(2)}\right]=} & {\left[\mathbb{H}^{(1)}, z_{1}+z_{2}\right]\left(\beta_{0}+\frac{1}{2} \mathbb{H}^{(1)}\right)+\left[\mathbb{H}^{(1)}, z_{12} \Delta^{(1)}\right], } \\
{\left[S_{+}^{(0)}, \mathbb{H}^{(3)}\right]=} & {\left[\mathbb{H}^{(1)}, z_{1}+z_{2}\right]\left(\beta_{1}+\frac{1}{2} \mathbb{H}^{(2)}\right)+\left[\mathbb{H}^{(2)}, z_{1}+z_{2}\right]\left(\beta_{0}+\frac{1}{2} \mathbb{H}^{(1)}\right) } \\
& +\left[\mathbb{H}^{(2)}, z_{12} \Delta^{(1)}\right]+\left[\mathbb{H}^{(1)}, z_{12} \Delta^{(2)}\right],
\end{aligned}
$$

so that the commutator $\left[S_{+}^{(0)}, \mathbb{H}^{(\ell)}\right]$ is expressed in terms of the lower order kernels, $\mathbb{H}^{(k)}$ and $\Delta^{(k)}$ with $k \leq \ell-1$. The kernel $\Delta^{(1)}$ was calculated in [9] and $\Delta^{(2)}$ in [12].

The first of the equations Eq. (2.15a) states that the LO evolution kernel commutes with canonical generators of the conformal transformations. As a consequence, the LO kernel $h^{(1)}(\alpha, \beta)$ can be written as a function of the conformal ratio and restored from the spectrum of LO anomalous dimensions.

The second equation, Eq. (2.15b), is a first-order inhomogeneous differential equation on the NLO evolution kernel $\mathbb{H}^{(2)}$. The solution is defined up to a solution of the corresponding homogeneous equation. This ambiguity is fixed by the requirement on the kernel to reproduce the correct anomalous dimensions. The straightforward calculation [11] to restore the kernel becomes too complicated for the NNLO kernel. Below we describe a different (algebraic) method used in [13].

\section{Similarity transformation}

The generators $S_{\alpha}$ in an interacting theory (2.12) involve the evolution kernel $\mathbb{H}(a)$ and additional contribution $\Delta(a)$ due to the conformal anomaly. It is convenient to separate these term by a 
similarity transformation

$$
\mathbb{H}=\mathrm{U}^{-1} \mathbf{H U}, \quad S_{ \pm, 0}=\mathrm{U}^{-1} \mathbf{S}_{ \pm, 0} \mathrm{U}
$$

The operators, $\mathbb{H}$ and $\mathbf{H}$, have the same spectrum. Note also that going over to the "boldface" operators can be thought of as a change of the renormalization scheme, $\left[\mathscr{O}\left(z_{1}, z_{2}\right)\right]_{U}=\mathrm{U}\left[\mathscr{O}\left(z_{1}, z_{2}\right)\right]_{\overline{\mathrm{MS}}}$. The new operator satisfies the RGE

$$
\left(M \partial_{M}+\beta(a) \partial_{a}+\mathbf{H}(a)-\beta(a) \partial_{a} \mathrm{U} \cdot \mathrm{U}^{-1}\right)\left[\mathscr{O}\left(z_{1}, z_{2}\right)\right]_{U}=0 .
$$

One can use this freedom to bring the generators $\mathbf{S}_{\alpha}$ into the "canonical" form

$$
\mathbf{S}_{-}=S_{-}^{(0)}, \quad \mathbf{S}_{0}=S_{0}^{(0)}+\left(\bar{\beta}(a)+\frac{1}{2} \mathbf{H}(a)\right), \quad \mathbf{S}_{+}=S_{+}^{(0)}+\left(z_{1}+z_{2}\right)\left(\bar{\beta}(a)+\frac{1}{2} \mathbf{H}(a)\right) .
$$

Looking for the operator $\mathrm{U}$ in the form

$$
\mathrm{U}=e^{\mathbb{X}}, \quad \mathbb{X}(a)=a \mathbb{X}^{(1)}+a^{2} \mathbb{X}^{(2)}+\ldots
$$

and assuming that $\left[S_{-}^{(0)}, \mathbb{X}^{(k)}\right]=\left[S_{0}^{(0)}, \mathbb{X}^{(k)}\right]=0$ we get the following equations on $\mathbb{X}^{(k)}$

$$
\begin{aligned}
& {\left[S_{+}^{(0)}, \mathbb{X}^{(1)}\right]=z_{12} \Delta^{(1)}} \\
& {\left[S_{+}^{(0)}, \mathbb{X}^{(2)}\right]=z_{12} \Delta^{(2)}+\left[\mathbb{X}^{(1)}, z_{1}+z_{2}\right]\left(\beta_{0}+\frac{1}{2} \mathbb{H}^{(1)}\right)+\frac{1}{2}\left[\mathbb{X}^{(1)}, z_{12} \Delta^{(1)}\right] .}
\end{aligned}
$$

Note, that these equations fix $\mathbb{X}^{(k)}$ up to $S L(2)$ invariant parts. In order words the transformation which brings the generators to the canonical form (3.3) is not unique. The one-loop kernel, $\mathbb{X}^{(1)}$, turns out to be rather simple

$$
\mathbb{X}^{(1)} f\left(z_{1}, z_{2}\right)=2 C_{F}\left(\int_{0}^{1} d \alpha \frac{\ln \alpha}{\alpha}\left[2 f\left(z_{1}, z_{2}\right)-f\left(z_{12}^{\alpha}, z_{2}\right)-f\left(z_{1}, z_{21}^{\alpha}\right)\right]\right)+\Delta \mathbb{X}_{\text {inv }}^{(1)} f\left(z_{1}, z_{2}\right) .
$$

The two-loop operator, $\mathbb{X}^{(2)}$ can be written as the sum of three terms

$$
\mathbb{X}^{(2)}=\mathbb{X}_{I}^{(2)}+\mathbb{X}^{(2,1)}\left(\beta_{0}+\frac{1}{2} \mathbb{H}^{(1)}\right)-\frac{1}{2} \mathbb{X}^{(2,2)}
$$

corresponding to the three contributions on the r.h.s. of Eq. (3.5), The explicit expressions for the kernels $\mathbb{X}_{\mathrm{I}}^{(2)}, \mathbb{X}^{(2,1)}, \mathbb{X}^{(2,2)}$ are quite involved and can be found in [13].

The operator $\mathbf{H}$ satisfies the set of equations (2.15c) with all terms containing the conformal anomaly $\Delta$ removed

$$
\begin{aligned}
& {\left[S_{+}^{(0)}, \mathbf{H}^{(1)}\right]=0,} \\
& {\left[S_{+}^{(0)}, \mathbf{H}^{(2)}\right]=\left[\mathbf{H}^{(1)}, z_{1}+z_{2}\right]\left(\beta_{0}+\frac{1}{2} \mathbf{H}^{(1)}\right),} \\
& {\left[S_{+}^{(0)}, \mathbf{H}^{(3)}\right]=\left[\mathbf{H}^{(1)}, z_{1}+z_{2}\right]\left(\beta_{1}+\frac{1}{2} \mathbf{H}^{(2)}\right)+\left[\mathbf{H}^{(2)}, z_{1}+z_{2}\right]\left(\beta_{0}+\frac{1}{2} \mathbf{H}^{(1)}\right) .}
\end{aligned}
$$


The solution can be written in the form

$$
\begin{aligned}
& \mathbf{H}^{(1)}=\mathbf{H}_{\text {inv }}^{(1)} \\
& \mathbf{H}^{(2)}=\mathbf{H}_{\text {inv }}^{(2)}+\mathbb{T}^{(1)}\left(\beta_{0}+\frac{1}{2} \mathbf{H}_{\text {inv }}^{(1)}\right), \\
& \mathbf{H}^{(3)}=\mathbf{H}_{\text {inv }}^{(3)}+\mathbb{T}^{(1)}\left(\beta_{1}+\frac{1}{2} \mathbf{H}_{\text {inv }}^{(2)}\right)+\mathbb{T}_{1}^{(2)}\left(\beta_{0}+\frac{1}{2} \mathbf{H}_{\text {inv }}^{(1)}\right)^{2}+\left(\mathbb{T}^{(2)}+\frac{1}{2}\left(\mathbb{T}^{(1)}\right)^{2}\right)\left(\beta_{0}+\frac{1}{2} \mathbf{H}_{\text {inv }}^{(1)}\right),
\end{aligned}
$$

where $\mathbf{H}_{\text {inv }}^{(k)}$ are (canonically) $S L(2)$-invariant operators with kernels that are functions of the conformal ratio and the operators $\mathbb{T}^{(i)}$ commute with $S_{-}^{(0)}$ and $S_{0}^{(0)}$ and obey the following equations:

$$
\left[S_{+}^{(0)}, \mathbb{T}^{(1)}\right]=\left[\mathbf{H}_{\mathrm{inv}}^{(1)}, z_{1}+z_{2}\right], \quad\left[S_{+}^{(0)}, \mathbb{T}^{(2)}\right]=\left[\mathbf{H}_{\mathrm{inv}}^{(2)}, z_{1}+z_{2}\right], \quad\left[S_{+}^{(0)}, \mathbb{T}_{1}^{(2)}\right]=\left[\mathbb{T}^{(1)}, z_{1}+z_{2}\right] .
$$

Similar to the $\mathbb{X}$ kernels the $\mathbb{T}$ kernels are fixed by Eqs. (3.10) up to $S L(2)$ (canonically) invariant terms. The $S L(2)$-invariant kernels $\mathbf{H}_{\text {inv }}^{(k)}$ can be written in the following general form

$$
\begin{aligned}
\mathbf{H}_{\text {inv }}^{(k)} f\left(z_{1}, z_{2}\right)= & \Gamma_{\text {cusp }}^{(k)} \int_{0}^{1} d \alpha \frac{\bar{\alpha}}{\alpha}\left(2 f\left(z_{1}, z_{2}\right)-f\left(z_{12}^{\alpha}, z_{2}\right)-f\left(z_{1}, z_{21}^{\alpha}\right)\right)+\chi_{0}^{(k)} f\left(z_{1}, z_{2}\right) \\
& +\int_{0}^{1} d \alpha \int_{0}^{\bar{\alpha}} d \beta\left(\chi_{\text {inv }}^{(k)}(\tau)+\chi_{\text {inv }}^{\mathbb{P}(k)}(\tau) \mathbb{P}_{12}\right) f\left(z_{12}^{\alpha}, z_{21}^{\beta}\right) .
\end{aligned}
$$

Here $z_{12}^{\alpha}=z_{1} \bar{\alpha}+z_{2} \alpha \tau=\alpha \beta /(\bar{\alpha} \bar{\beta})$ and $\mathbb{P}_{12}$ is the permutation operator, $\mathbb{P}_{12} f\left(z_{1}, z_{2}\right)=f\left(z_{2}, z_{1}\right)$. $\Gamma_{\text {cusp }}$ is the cusp anomalous dimension which is known to the required accuracy [1]. The LO expression for the $\chi$-functions corresponds to

$$
\chi_{0}^{(1)}=2 C_{F}, \quad \chi_{\text {inv }}^{(1)}(\tau)=-4 C_{F}, \quad \chi_{\text {inv }}^{\mathbb{P}(1)}(\tau)=0 .
$$

and

$$
\begin{aligned}
\chi_{0}^{(2)} & =\frac{1}{3} C_{F}\left\{\beta_{0}\left(37-4 \pi^{2}\right)+C_{F}\left(43-4 \pi^{2}\right)+\frac{1}{N_{c}}\left(26-8 \pi^{2}+72 \zeta_{3}\right)\right\}, \\
\chi_{\mathrm{inv}}^{(2)}(\tau) & =4 C_{F}\left\{-\frac{11}{3} \beta_{0}+C_{F}\left[\ln \bar{\tau}-\frac{20}{3}+\frac{2 \pi^{2}}{3}\right]-\frac{2}{N_{c}}\left(\mathrm{Li}_{2}(\tau)+\frac{1}{2} \ln ^{2} \bar{\tau}-\frac{1}{\tau} \ln \bar{\tau}-\frac{\pi^{2}}{6}+\frac{5}{3}\right)\right\}, \\
\chi_{\mathrm{inv}}^{\mathbb{P}(2)}(\tau) & =-\frac{4 C_{F}}{N_{c}}\left(\ln ^{2} \bar{\tau}-2 \tau \ln \bar{\tau}\right) .
\end{aligned}
$$

The corresponding expressions for the $\mathbb{T}$ kernels take the form:

$$
\begin{aligned}
\mathbb{T}^{(k)} f\left(z_{1}, z_{2}\right)= & -\Gamma_{\text {cusp }}^{(k)} \int_{0}^{1} d \alpha \frac{\bar{\alpha} \ln \bar{\alpha}}{\alpha}\left(f\left(z_{12}^{\alpha}, z_{2}\right)+f\left(z_{1}, z_{21}^{\alpha}\right)\right) \\
& +\int_{0}^{1} d \alpha \int_{0}^{\bar{\alpha}} d \beta \ln (1-\alpha-\beta)\left(\chi_{\text {inv }}^{(k)}(\tau)+\chi_{\text {inv }}^{\mathbb{P}(k)}(\tau) \mathbb{P}_{12}\right) f\left(z_{12}^{\alpha}, z_{21}^{\beta}\right),
\end{aligned}
$$

and

$$
\mathbb{T}_{1}^{(2)} f\left(z_{1}, z_{2}\right)=-\int_{0}^{1} d \alpha \frac{\bar{\alpha} \ln ^{2} \bar{\alpha}}{2 \alpha}\left[f\left(z_{12}^{\alpha}, z_{2}\right)+f\left(z_{1}, z_{21}^{\alpha}\right)\right]+\int_{0}^{1} d \alpha \int_{0}^{\bar{\alpha}} d \beta \frac{\ln ^{2}(1-\alpha-\beta)}{2} f\left(z_{12}^{\alpha}, z_{21}^{\beta}\right) .
$$


Equations (3.14), (3.15) together with the expression for the kernel $\mathbb{X}$ fix the noninvariant part part of the NNLO evolution kernel (with respect to the canonical generators $S_{\alpha}^{(0)}$ )

$$
\begin{aligned}
\mathbb{H}^{(1)}= & \mathbf{H}^{(1)}=\mathbf{H}_{\mathrm{inv}}^{(1)}, \\
\mathbb{H}^{(2)}= & \mathbf{H}^{(2)}+\left[\mathbf{H}^{(1)}, \mathbb{X}^{(1)}\right]=\mathbf{H}_{\mathrm{inv}}^{(2)}+\mathbb{T}^{(1)}\left(\beta_{0}+\frac{1}{2} \mathbf{H}_{\mathrm{inv}}^{(1)}\right)+\left[\mathbf{H}_{\mathrm{inv}}^{(1)}, \mathbb{X}^{(1)}\right] \\
\mathbb{H}^{(3)}= & \mathbf{H}^{(3)}+\left[\mathbf{H}^{(2)}, \mathbb{X}^{(1)}\right]+\left[\mathbf{H}^{(1)}, \mathbb{X}^{(2)}\right]+\frac{1}{2}\left\{\mathbf{H}^{(2)},\left(\mathbb{X}^{(1)}\right)^{2}\right\} \\
= & \mathbf{H}_{\mathrm{inv}}^{(3)}+\mathbb{T}^{(1)}\left(\beta_{1}+\frac{1}{2} \mathbf{H}_{\mathrm{inv}}^{(2)}\right)+\mathbb{T}_{1}^{(2)}\left(\beta_{0}+\frac{1}{2} \mathbf{H}_{\mathrm{inv}}^{(1)}\right)^{2}+\left(\mathbb{T}^{(2)}+\frac{1}{2}\left(\mathbb{T}^{(1)}\right)^{2}\right)\left(\beta_{0}+\frac{1}{2} \mathbf{H}_{\mathrm{inv}}^{(1)}\right) \\
& +\left[\mathbf{H}_{\mathrm{inv}}^{(2)}, \mathbb{X}^{(1)}\right]+\frac{1}{2}\left[\mathbb{T}^{(1)} \mathbf{H}_{\mathrm{inv}}^{(1)}, \mathbb{X}^{(1)}\right]+\frac{1}{2}\left[\mathbf{H}_{\mathrm{inv}}^{(1)}, \mathbb{X}^{(2,1)}\right] \mathbf{H}_{\mathrm{inv}}^{(1)}+\left[\mathbf{H}_{\mathrm{inv}}^{(1)}, \mathbb{X}_{\mathrm{I}}^{(2)}\right] \\
& +\beta_{0}\left(\left[\mathbf{T}_{\mathrm{inv}}^{(1)}, \mathbb{X}^{(1)}\right]+\left[\mathbf{H}_{\mathrm{inv}}^{(1)}, \mathbb{X}^{(2,1)}\right]\right)+\frac{1}{2}\left[\left[\mathbf{H}_{\mathrm{inv}}^{(1)}, \mathbb{X}^{(1)}\right], \mathbb{X}^{(1)}\right]-\frac{1}{2}\left[\mathbf{H}_{\mathrm{inv}}^{(1)}, \mathbb{X}^{(2,2)}\right],
\end{aligned}
$$

Here all entries are known except for the $S L(2)$-invariant part of the three-loop kernel $\mathbf{H}_{\text {inv }}^{(3)}$. It has to be determined from the three loop anomalous dimensions [1].

\section{Three-loop invariant kernel $\mathbf{H}_{\text {inv }}^{(3)}$ and Gribov - Lipatov reciprocity relation}

The three-loop invariant kernel $\mathbf{H}_{\text {inv }}^{(3)}$ takes the form

$$
\begin{aligned}
\mathbf{H}_{\text {inv }}^{(3)} f\left(z_{1}, z_{2}\right)= & \Gamma_{\text {cusp }}^{(3)} \int_{0}^{1} d \alpha \frac{\bar{\alpha}}{\alpha}\left(2 f\left(z_{1}, z_{2}\right)-f\left(z_{12}^{\alpha}, z_{2}\right)-f\left(z_{1}, z_{21}^{\alpha}\right)\right)+\chi_{0}^{(3)} f\left(z_{1}, z_{2}\right) \\
& +\int_{0}^{1} d \alpha \int_{0}^{\bar{\alpha}} d \beta\left(\chi_{\text {inv }}^{(3)}(\tau)+\chi_{\text {inv }}^{\mathbb{P}(3)}(\tau) \mathbb{P}_{12}\right) f\left(z_{12}^{\alpha}, z_{21}^{\beta}\right) .
\end{aligned}
$$

The cusp anomalous dimension $\Gamma_{\text {cusp }}^{(3)}$ is known with three-loop accuracy [1]. Thus we have to determine the constant $\chi_{0}^{(3)}$ and two functions of one variable, $\chi_{\text {inv }}^{(3)}(\tau)$ and $\chi_{\text {inv }}^{\mathbb{P}(3)}(\tau)$. This can be achieved by using the information on the spectrum of the invariant kernel $\mathbf{H}_{\text {inv }}^{(3)}$

$$
\mathbf{H}_{\mathrm{inv}}^{(3)}\left(z_{1}-z_{2}\right)^{N}=\gamma_{\mathrm{inv}}^{(3)}(N)\left(z_{1}-z_{2}\right)^{N} .
$$

Making use of Eqs. (3.9) and (3.14), (3.15) it can be shown that

$$
\begin{aligned}
\gamma_{\text {inv }}^{(3)}(N)= & \gamma^{(3)}(N)-\left(\beta_{1}+\frac{1}{2} \gamma^{(2)}(N)\right) \frac{d}{d N} \gamma^{(1)}(N)-\left(\beta_{0}+\frac{1}{2} \gamma^{(1)}(N)\right) \frac{d}{d N} \gamma^{(2)}(N) \\
& +\frac{1}{2}\left(\beta_{0}+\frac{1}{2} \gamma^{(1)}(N)\right)\left(\frac{d}{d N} \gamma^{(1)}(N)\right)^{2}
\end{aligned}
$$

The anomalous dimensions $\gamma_{\text {inv }}^{(3)}(N)$ can be promoted to a function of the complex variable $N$. As usual it has to be done separately for even and odd values of $N$

$$
\gamma_{\text {inv }}^{(3)}(N)=\gamma_{\text {inv }}^{(3+)}(N)+(-1)^{N} \gamma_{\text {inv }}^{(3-)}(N)
$$


It can be shown [13] that the splitting functions corresponding to the anomalous dimensions $\gamma_{\text {inv }}^{ \pm}$ satisfy the Gribov-Lipatov reciprocity relation [16]

$$
\gamma_{\mathrm{inv}}^{(3 \pm)}(N)=-\int_{0}^{1} d x x^{N} H_{\mathrm{inv}}^{(3 \pm)}(x), \quad H_{\mathrm{inv}}^{(3 \pm)}(x)=-x H_{\mathrm{inv}}^{(3 \pm)}(1 / x) .
$$

The kernels $\chi, \chi^{\mathbb{P}}$ are completely determined by the anomalous dimensions and can be restored as follows [11]

$$
\begin{aligned}
\chi_{\mathrm{inv}}^{(3)}(\tau) & =\frac{1}{2 \pi i} \int_{c-i \infty}^{c+i \infty} d N(2 N+3) \Delta \gamma_{\mathrm{inv}}^{(3+)}(N) P_{N+1}\left(\frac{1+\tau}{1-\tau}\right), \\
\chi_{\mathrm{inv}}^{\mathbb{P}(3)}(\tau) & =\frac{1}{2 \pi i} \int_{c-i \infty}^{c+i \infty} d N(2 N+3) \gamma_{\mathrm{inv}}^{(3-)}(N) P_{N+1}\left(\frac{1+\tau}{1-\tau}\right),
\end{aligned}
$$

where

$$
\Delta \gamma_{\text {inv }}^{(3+)}(N)=\gamma_{\text {inv }}^{(3+)}(N)-2 \Gamma_{\text {cusp }}^{(3)}[\psi(N+2)-\psi(2)]-\chi_{0}^{(3)}
$$

and $P_{N+1}$ is the Legendre function. All singularities of the anomalous dimensions have to lie to the left of the integration contour. The representation (4.6) is not very practical since three loop anomalous dimensions are expressed in terms of the harmonic sums whose analytic properties are not well studied. However, starting from Eqs. (4.6) one can obtain a representation for the kernels in terms of the corresponding splitting functions. Namely,

$$
\chi(\tau)=-\frac{1}{2 \pi i} \int_{c-i \infty}^{c+i \infty} d \rho\left(\frac{\tau}{\bar{\tau}}\right)^{\rho} \frac{\Gamma(2 \rho+2)}{\Gamma^{2}(1+\rho)} \int_{0}^{1} d x H(x) \frac{1}{x \bar{x}}\left(\frac{1+x}{1-x}\right)\left(\frac{x}{\bar{x}^{2}}\right)^{\rho} .
$$

We cannot calculate this integral analytically for exact splitting functions. However, one can try to use some approximation for $H$. We use a parametrization for the splitting functions consistent with the reciprocity relations and separate the leading contributions at $x \rightarrow 0$ and $x \rightarrow 1$

$$
\begin{aligned}
\Delta H_{\mathrm{inv}}^{(3+)}(x) & =\sum_{k=1}^{4} B_{k}^{(3+)} \phi_{k}(x)+\bar{x} C_{0}^{(3+)}+\bar{x} C_{1}^{(3+)} \ln \left(\frac{x}{\bar{x}^{2}}\right)+\delta H_{\mathrm{inv}}^{(3+)}(x), \\
H_{\mathrm{inv}}^{(3-)}(x) & =\sum_{k=1}^{4} B_{k}^{(3-)} \phi_{k}(x)+\bar{x} C_{0}^{(3-)}+\delta H_{\mathrm{inv}}^{(3-)}(x) .
\end{aligned}
$$

The functions $\phi_{k}$ are defined recursively:

$$
\phi_{0}(x)=1-x, \quad \phi_{k}(x)=\int_{x}^{1} \frac{d \xi}{\xi} \phi_{k-1}(\xi) \phi_{0}(x / \xi), \quad \int_{0}^{1} d x x^{j-2} \phi_{k}(x)=\left(\frac{1}{j(j-1)}\right)^{k+1}
$$

The coefficients $B_{k}^{(3 \pm)}, C_{k}^{3 \pm}$ can be extracted from the expression for the splitting functions and the remainder functions $\delta H_{\text {inv }}^{(3 \pm)}(x)$ we parameterize as follows

$$
\delta H_{\mathrm{inv}}^{(3 \pm)}(x)=\bar{x} h_{ \pm}\left(x / \bar{x}^{2}\right), \quad h_{ \pm}(t)=H_{0}^{ \pm} \frac{a_{ \pm}}{t+a_{ \pm}}\left(1+\frac{b_{ \pm} t}{t+a_{ \pm}}\right) .
$$




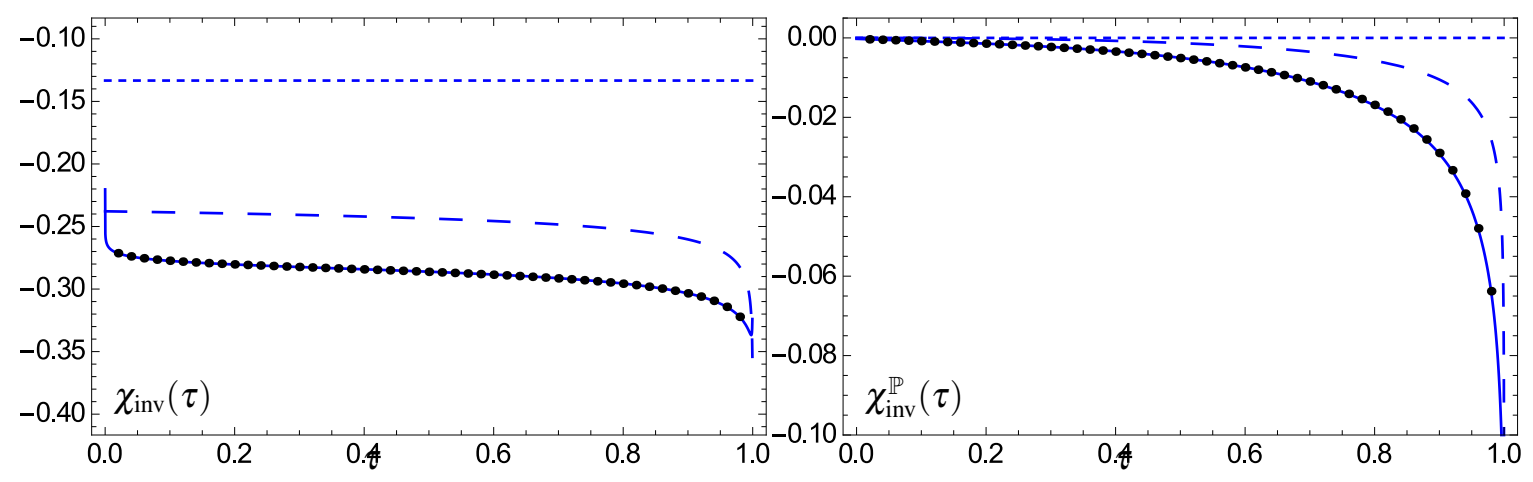

Figure 1: Invariant functions $\chi_{\text {inv }}(\tau)$ (left panel) and $\chi_{\text {inv }}^{\mathbb{P}}(\tau)$ (right panel) for $\alpha_{s} / \pi=0.1$. The LO result (short dashes) is shown together with the NLO (long dashes) and NNLO (solid curves). The NNLO results using exact $\mathscr{O}\left(a^{3}\right)$ functions obtained by the numerical integration of Eq. (4.6) are shown by black dots for comparison.

With this simple parametrization one can achieve the accuracy $\left(\left.\delta H_{\text {inv }}^{(3 \pm)}\right|_{\text {fit }}-\left.\delta H_{\text {inv }}^{(3 \pm)}\right|_{\text {exact }}\right) / H_{\text {inv }}^{(3 \pm)}<$ $0.5 \%$ in the whole range of $x$. The numerical values for the parameters of the fit can be found in [13].

The integrals (4.8) with the splitting functions (4.9) can be calculated analytically. We show in Fig. 1 the full NNLO invariant functions $\chi(a)=a \chi^{(1)}+a^{2} \chi^{(2)}+a^{3} \chi^{(3)}$ together with the NLO, $\mathscr{O}\left(a^{2}\right)$, and the LO, $\mathscr{O}(a)$, ones for a typical value of the coupling $\alpha_{s} / \pi=0.1$ and for definiteness, $n_{f}=4$. The exact NNLO results obtained by the numerical integration of Eq. (4.6) are shown by dots. One sees that the accuracy of the parametrization is rather good. The remaining entries in the invariant kernel, (Eq. (3.11)) for the same values of flavors and coupling constant, are

$$
\begin{aligned}
\Gamma_{\text {cusp }} & =a \Gamma^{(1)}\left(1+8.019 a+80.53 a^{2}+\ldots\right)=a \Gamma^{(1)}(1+0.2005+0.0503+\ldots), \\
\chi_{0} & =a \chi_{0}^{(1)}\left(1-0.7935 a-141.3 a^{2}+\ldots\right)=a \chi_{0}^{(1)}(1-0.0198-0.0883+\ldots) .
\end{aligned}
$$

\section{Summary}

The evolution equation for the flavor non-singlet leading twist operators is known now with three loop accuracy $[12,13]$. The non-invariant part of the evolution kernel was obtained in an explicit form while for the invariant part a rather accurate approximation was developed. The matrix of the anomalous dimensions for the local operators up to dimension $D \leq 10$ was also presented [13]. These results can be used for studying the scale dependence of generalized parton distributions, see e.g. $[17,18]$, or light-cone meson distribution amplitudes.

\section{Acknowledgments}

The author thanks V. M. Braun, S. Moch and M. Strohmaier for the collaboration on this project. The study was supported by Deutsche Forschungsgemeinschaft (DFG) with the grant MO 1801/1-2. 


\section{References}

[1] S. Moch, J. A. M. Vermaseren and A. Vogt, The Three loop splitting functions in QCD: The Nonsinglet case, Nucl. Phys. B688 (2004) 101-134, [hep-ph/ 0403192$].$

[2] A. Vogt, S. Moch and J. A. M. Vermaseren, The Three-loop splitting functions in QCD: The Singlet case, Nucl. Phys. B691 (2004) 129-181, [hep-ph/ 0404111$].$

[3] D. Mueller, Constraints for anomalous dimensions of local light cone operators in phi**3 in six-dimensions theory, Z. Phys. C49 (1991) 293-300.

[4] D. Mueller, Conformal constraints and the evolution of the nonsinglet meson distribution amplitude, Phys. Rev. D49 (1994) 2525-2535.

[5] D. Mueller, Restricted conformal invariance in $Q C D$ and its predictive power for virtual two photon processes, Phys. Rev. D58 (1998) 054005, [hep-ph/9704406].

[6] A. V. Belitsky and D. Mueller, Predictions from conformal algebra for the deeply virtual Compton scattering, Phys. Lett. B417 (1998) 129-140, [hep-ph/9709379].

[7] A. V. Belitsky and D. Mueller, Next-to-leading order evolution of twist-2 conformal operators: The Abelian case, Nucl. Phys. B527 (1998) 207-234, [hep-ph/9802411].

[8] A. V. Belitsky, A. Freund and D. Mueller, Evolution kernels of skewed parton distributions: Method and two loop results, Nucl. Phys. B574 (2000) 347-406, [hep-ph/9912379].

[9] A. V. Belitsky and D. Mueller, Broken conformal invariance and spectrum of anomalous dimensions in QCD, Nucl. Phys. B537 (1999) 397-442, [hep-ph/ 9804379$].$

[10] V. M. Braun and A. N. Manashov, Evolution equations beyond one loop from conformal symmetry, Eur. Phys. J. C73 (2013) 2544, [1306.5644].

[11] V. M. Braun and A. N. Manashov, Two-loop evolution equations for light-ray operators, Phys. Lett. B734 (2014) 137-143, [1404.0863].

[12] V. M. Braun, A. N. Manashov, S. Moch and M. Strohmaier, Two-loop conformal generators for leading-twist operators in QCD, JHEP 03 (2016) 142, [1 601 . 05937].

[13] V. M. Braun, A. N. Manashov, S. Moch and M. Strohmaier, Three-loop evolution equation for flavor-nonsinglet operators in off-forward kinematics, JHEP 06 (2017) 037, [1 703.09532 ].

[14] I. I. Balitsky and V. M. Braun, Evolution Equations for QCD String Operators, Nucl. Phys. B311 (1989) 541-584.

[15] V. M. Braun, S. E. Derkachov, G. P. Korchemsky and A. N. Manashov, Baryon distribution amplitudes in QCD, Nucl. Phys. B553 (1999) 355-426, [hep-ph/9902375].

[16] V. N. Gribov and L. N. Lipatov, e+e-pair annihilation and deep inelastic e p scattering in perturbation theory, Sov. J. Nucl. Phys. 15 (1972) 675-684.

[17] D. Mueller, Generalized Parton Distributions - visions, basics, and realities -, Few Body Syst. 55 (2014) 317-337, [1405.2817].

[18] D. Mueller, Uses of $Q^{* * 2}$ evolution in GPD phenomenology, Int. J. Mod. Phys. Conf. Ser. 04 (2011) $168-178$. 